\title{
Trigonometric Functions at a Crossroads
}

\author{
Jiří Stávek ${ }^{1}$ \\ ${ }^{1}$ Bazovského 1228, 16300 Prague, Czech republic \\ Correspondence: Jiří Stávek, Bazovského 1228, 16300 Prague, Czech republic. E-mail: stavek.jiri@seznam.cz \\ Received: May 9, 2017 \\ Accepted: May 22, 2017 \\ Online Published: May 31, 2017 \\ doi:10.5539/apr.v9n3p40 \\ URL: https://doi.org/10.5539/apr.v9n3p40
}

\begin{abstract}
In the history of physics trigonometric functions played several times a very critical role at crossroads. This time we are at a crossroads with the interpretation of correlation events of entangled particles. In this approach we propose to describe the experimental data of Alice and Bob using not so known trigonometric functions. Claudius Theorem (based on the trigonometric family of Sagitta and Cosagitta) evalutes the probabilistic occurrence of correlated and anticorrelated events. David Theorem (based on the trigonometric family of Hacoversine) describes the probability of the following identical events and gaps between the following identical events. In this trigonometric concept the Team of Alice, Bob, Claudius and David formulated a camouflage legend for Eve "spooky action at a distance". Merlin (with unbounded computational ability) should verify the truth of this statement. Trent (a trusted arbitrator, who acts as a neutral third party) should analyze these data and this trigonometric concept. Victor (a verifier) should make his decision which way we should continue in our future research: either through the Niels Bohr avenue or through the Albert Einstein sidewalk.
\end{abstract}

Keywords: Trigonometric functions at a crossroads, Sagitta, Cosagitta, Hacoversine, a camouflage legend.

\section{Introduction}

The application of trigonometric functions played several times in the history of physics a very critical role. The analysis of experimental data with a chosen trigonometric function and its known precise value at that time determined the model explaining those phenomena.

E.g., Claudius Ptolemy described the experimental data of planet motions using the trigonometric function chord (at his time the only trigonometric function developed earlier by Hipparchus). During the following 1400 years trigonometric functions were intensively studied by Old Masters - the historical overview of the development of trigonometry can be found in the works of Von Braumühl (1900), Datta and Singh (1983), Matvievskaya (1990), Maor (1998), Van Brumelen (2009, 2013, 2014), and Smýkalová (2015). Johann Müller (called Regiomantus, 1436 - 1476) summarized the knowledge about trigonometric functions in his book "De triangulis Omnimodis" (On Triangles of Every Kind). This book served as "the foot of the ladder to the stars" and inspired Copernicus, Rheticus, Brahe, Kepler and many others. Nicolaus Copernicus created his model using the trigonometric function sine. Georg Rheticus $(1514$ - 1574) continued to further develop trigonometric functions in his "Opus Palatinum de Triangulis" (Canon of the Science of Triangles) which became the first printed publication of tables of all six trigonometric functions. Johannes Kepler analyzed the experimental data of Tycho Brahe using these more precise trigonometric functions and discovered the elliptical paths of planets around the Sun.

Today, we are again at a crossroads: we have available very precise experimental data of correlations of entangled particles that are excellently described by the trigonometric functions derived by the Quantum Mechanics. However, in this case we have to give up the concept of the local realism.

The latest experiments of leaders in this field closed all experimental loopholes for the local realism: Jan-Åke Larsson in 2014, Marissa Gustina et al. in 2015, Lynden K. Shalm et al. in 2015, Bradley G. Christensen et al. in 2015, Alain Aspect in 2015, Johannes Handsteiner et al. in 2017, Reinhold Bertlmann and Anton Zeilinger in 2017. Andrei Khrennikov organized in June $13-16,2016$ a great meeting with presentations about the state of the art in this field - see the video presentations on his website. Amir D. Aczel (2001) surveyed the complexity of this long research.

It seems it makes no sence to continue to protect the local realism. However, there remains one hidden door leading to the local realism - the realm of trigonometric functions. We have still a possibility to describe the correlation events of entangled particles by less known (partly forgotten and partly not yet discovered) trigonometric functions. 
In the first stage we have to describe the known experimental data of Alice and Bob by some other trigonometric approach as was done by the Quantum Mechanics. For this case we will apply the Claudius Theorem based on the trigonometric family Sagitta and Cosagitta that describes probabilistically numbers of correlated and anticorrelated events. However, this Claudius Theorem does not meet the requirement of the Karl Popper's concept of the falsification of data (1959) because it only reproduces the concept of Max Born - the Born rule. Moreover, we have to fulfill two conditions of Werner Heisenberg and Albert Einstein. Werner Heisenberg stated that any good theory must be based only on directly observable magnitudes. On the other hand Albert Einstein stated that it is the a good theory which decides what we can observe. We have to discover a new trigonometric function for the falsification of the existing concept and its interpretation. For this purpose we will use the David Theorem based on the trigonometric family of Hacoversine that enables newly to describe correlation events. In this case we will quantify the gaps between the following identical events. This concept could be accepted both by Werner Heisenberg and Albert Einstein.

In this trigonometric scenario the Team of Alice, Bob, Claudius and David formulate a camouflage legend for Eve - "spooky action at a distance". Merlin (with unbounded computational ability) should verify the truth of this statement. Trent (a trusted arbitrator, who acts as a neutral third party) should analyze these data and this trigonometric concept. Victor (a verifier) should make his decision which way we should continue in our future research: either through the Niels Bohr avenue or through the Albert Einstein sidewalk.

\section{Trigonometric Functions in Circles with Radius $R=1, R=1 / 2$, and $R=1 / 4$}

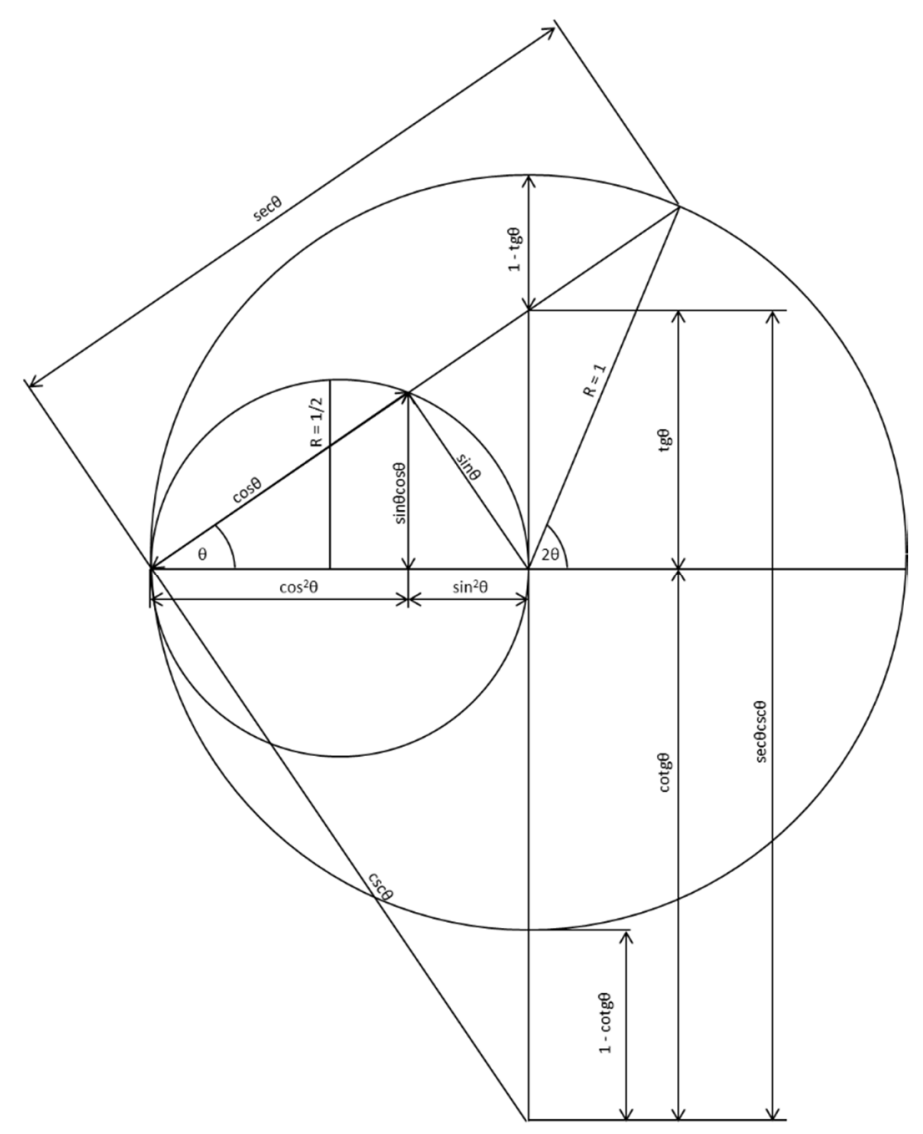

Figure 1 Three Rheticus triangles in the circles with radius $\mathrm{R}=1$ and $\mathrm{R}=1 / 2$ - the realm of trigonometric functions

The development of trigonometric functions has a very rich and long history. This knowledge was flowing from the ancient Egyptians, Babylonians and Greeks through India and Arabic countries back to Europe. The Old Masters related trigonometric functions to arcs of circles and lengths of chords subtending their arcs. Regiomantus determined values from right-triangle ratios. Modern scholars have been using mostly the unit circle with radius $\mathrm{R}=1$ for the analysis of properties of the main six trigonometric functions. 


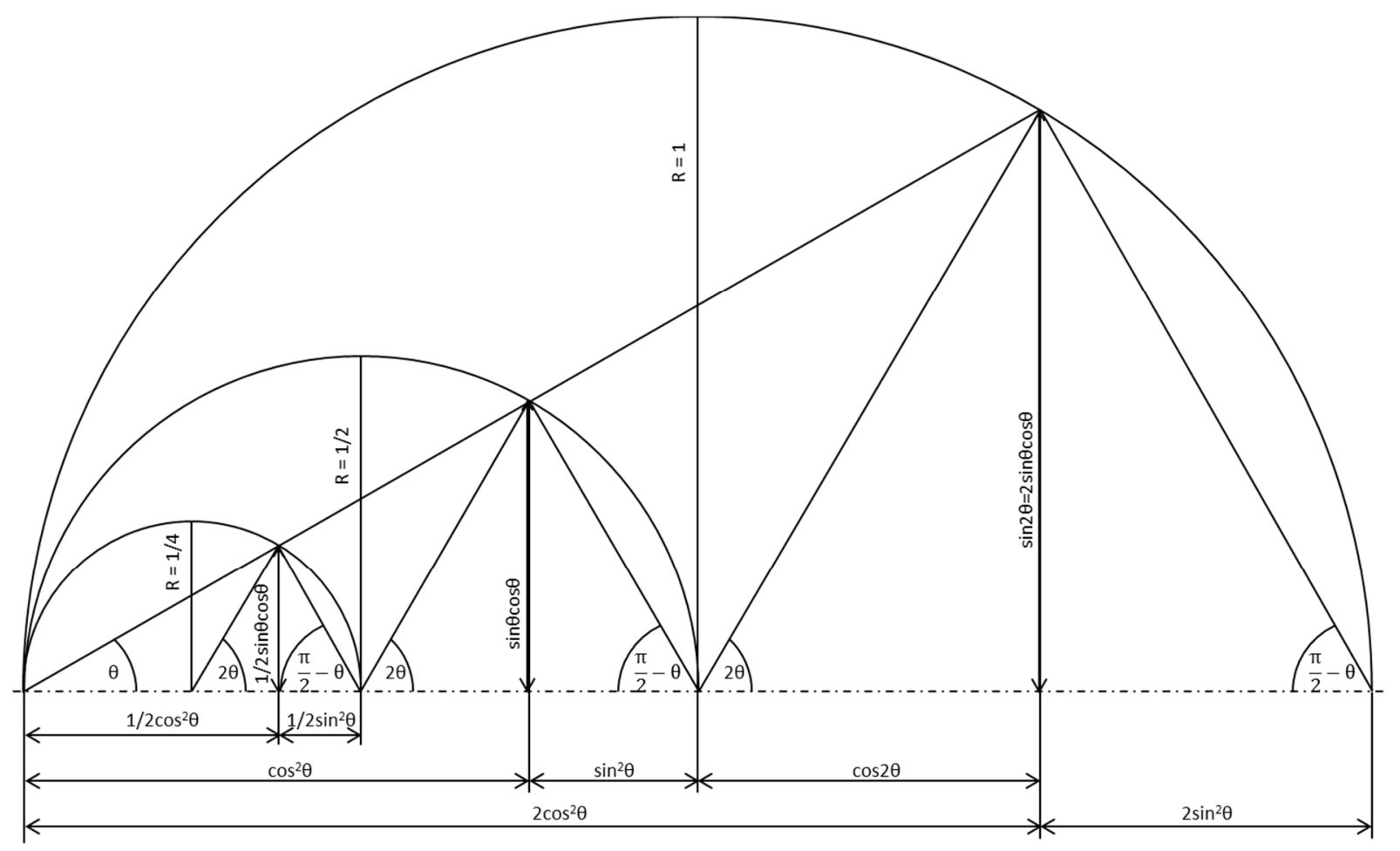

Figure 2. Trigonometric functions in circles with radius $\mathrm{R}=1, \mathrm{R}=1 / 2$ and $\mathrm{R}=1 / 4$

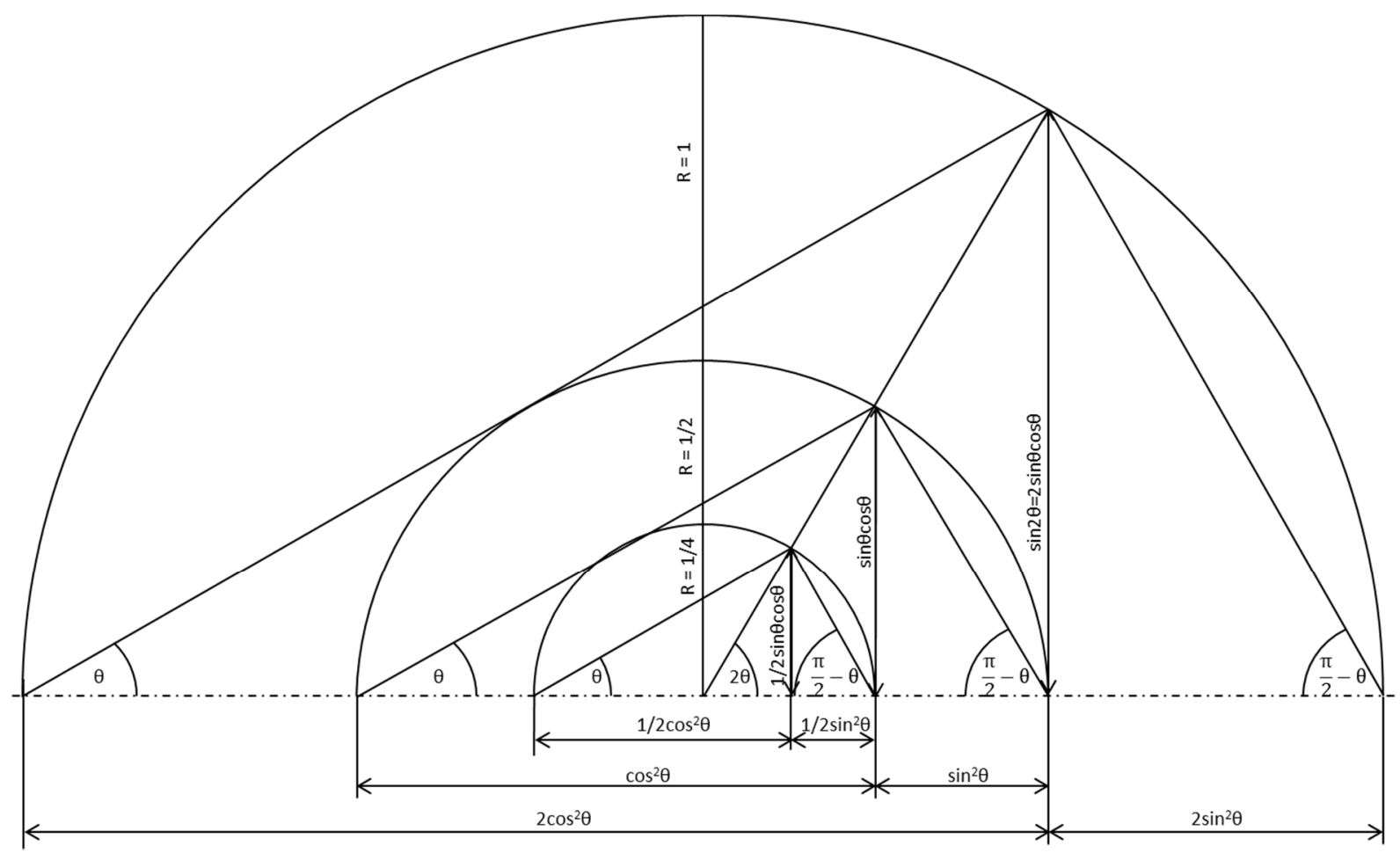

Figure 3. Orbits with radius $\mathrm{R}=1, \mathrm{R}=1 / 2$ and $\mathrm{R}=1 / 4$ and the trigonometric functions

Galileo gave to us his advice in Opere Il Saggiatore p. 171: "[The Universe] cannot be read until we have learnt the language and become familiar with the characters in which it is written. It is written in mathematical language, and the letters are triangles, circles and other geometrical figures, without which means it is humanly impossible to comprehend a single word." 
This Galileo's advice inspired us on one side to describe those circles and triangles with the existing known trigonometric relations, and on the other side to discover some new trigonometric relations that might be useful for the description of correlation events among entangled particles.

In our concept we are searching for the new trigonometric functions in circles with radius $R=1 / 2$ and $R=1 / 4$. In these circles we have found several new trigonometric functions that might be useful for the description of correlations among the entangled particles.

In Figure 1 we can see an opened door to the realm of trigonometric functions that prepared for us Georg Rheticus in his book "Canon of the Science of Triangles" in 1551 - see the video lecture of Glen van Brummelen in 2014 and the Figure 2.2. in Radka Smýkalová (2015).

Figure 2 and Figure 3 show circles with the radius $R=1, R=1 / 2$ and $R=1 / 4$ and several triangles that reveal many trigonometric functions hidden in those lines. We will focuse our attention on trigonometric families Sagitta, Cosagitta and Hacoversine.

\section{Claudius Theorem and David Theorem}

Table 1. Claudius Theorem describing the total probabilities of joint detections and David Theorem describing probabilities of the following identical events and gaps between them

\begin{tabular}{|c|c|c|c|}
\hline \multicolumn{4}{|c|}{ Claudius Theorem - probabilistic occurence of joint detections } \\
\hline $\mathrm{P}++$ & $\mathrm{P}+-$ & $\mathrm{P}-+$ & P-- \\
\hline$\frac{1}{2} \cos ^{2} \theta$ & $\frac{1}{2} \sin ^{2} \theta$ & $\frac{1}{2} \cos ^{2}\left(\frac{\pi}{2}-\theta\right)$ & $\frac{1}{2} \sin ^{2}\left(\frac{\pi}{2}-\theta\right)$ \\
\hline$\frac{1}{2} \cos ^{2} \theta$ & $\frac{1}{2} \sin ^{2} \theta$ & $\frac{1}{2} \sin ^{2} \theta$ & $\frac{1}{2} \cos ^{2} \theta$ \\
\hline \multicolumn{4}{|c|}{ David Theorem - probabilities of the following identical detections } \\
\hline $\mathrm{P}++\leftrightarrow \mathrm{P}++$ & $\mathrm{P}+-\leftrightarrow \mathrm{P}+-$ & $\mathrm{P}-+\leftrightarrow \mathrm{P}-+$ & P-- $\leftrightarrow$ P-- \\
\hline$\frac{1}{2}-\frac{1}{2} \sin \theta \cos \theta$ & $\frac{1}{2} \sin \theta \cos \theta$ & $\frac{1}{2} \sin \left(\frac{\pi}{2}-\theta\right) \cos \left(\frac{\pi}{2}-\theta\right.$ & $\frac{1}{2}-\frac{1}{2} \sin \left(\frac{\pi}{2}-\theta\right) \cos \left(\frac{\pi}{2}-\theta\right.$ \\
\hline$\frac{1}{2}-\frac{1}{2} \sin \theta \cos \theta$ & $\frac{1}{2} \sin \theta \cos \theta$ & $\frac{1}{2} \cos \theta \sin \theta$ & $\frac{1}{2}-\frac{1}{2} \cos (\theta) \sin (\theta)$ \\
\hline \multicolumn{4}{|c|}{ David Theorem - gaps between identical detections } \\
\hline $\mathrm{P}++\leftrightarrow \mathrm{P}++$ & $\mathrm{P}+-\leftrightarrow \mathrm{P}+-$ & $\mathrm{P}-+\leftrightarrow \mathrm{P}-+$ & P-- $\leftrightarrow$ P-- \\
\hline$\frac{2}{1-\sin \theta \cos \theta}$ & $\frac{2}{\sin \theta \cos \theta}$ & $\frac{2}{\sin \left(\frac{\pi}{2}-\theta\right) \cos \left(\frac{\pi}{2}-\theta\right)}$ & $\frac{2}{1-\sin \left(\frac{\pi}{2}-\theta\right) \cos \left(\frac{\pi}{2}-\theta\right)}$ \\
\hline$\frac{2}{1-\sin \theta \cos \theta}$ & $\frac{2}{\sin \theta \cos \theta}$ & $\frac{2}{\cos \theta \sin \theta}$ & $\frac{2}{1-\cos \theta \sin \theta}$ \\
\hline
\end{tabular}

The mathematical language of Quantum Mechanics perfectly describes probabilistically correlation events among entangled particles. This is the reason why most of researchers stated that this mathematical language completely characterizes those correlation events and that there is no hope to find anything better. A minority of researchers 
tries to discover another mathematical language that could bring more information from the microworld of correlated particles and to protect the local realism.

We propose to apply two Theorems for the description of correlation events among entagled particles:

1) Claudius Theorem describes the probabilities of joint correlated or anticorrelated detections. The hidden microworld sends to us these signals that are described by symmetric trigonometric functions Sagitta and Cosagitta. It could be a very clever trick of Nature to create a camouflage legend for Eve as "spooky action at a distance". Eve's knowledge based on the mathematical language of Quantum Mechanics has to come to this camouflage legend. This trigonometric model documents that Nature has a great hiding power to protect Her secrets.

2) David Theorem brings a new additional concept for the description of the correlation of entangled particles. David (see the statue of Michelangelo) proposed to measure gaps between the following identical events in the SPDC Type I process where horizontally and vertically pairs have been created.

Table 1 summarizes our trigonometric model for the SPDC Type I (spontaneous parametric down-conversion type I) where pairs of entangled photons are created either horizontally or vertically polarized. The predictions of this David Theorem can be easily experimentally tested in the Laboratories of Leaders in this field. The probabilistic terminology P++, P+-, P-+, P-- describing the correlated and anticorrelated "clicks" was inspired by Alain Aspect.

Both Quantum Mechanics and Trigonometric Mechanics cannot with certainty predict the outcome of all single events, but instead they predict probabilities of outcomes. The mathematical language of Quantum Mechanics does not protect the local realism. The mathematical language of Trigonometric Mechanics protects the local realism. In the Trigonometric Mechanics quantum systems are probabilistically controlled by "hidden variables" coming form the realm of trigonometric functions that determine the outcomes of measurements.

Ladislav Kvasz (2008) discussed how the mathematical language influences the interpretation of observed phenomena.

\section{Conclusions}

1) In the circles with radius $R=1 / 2$ and $R=1 / 4$ several less known trigonometric functions were found and several new trigonometric relations were discovered.

2) Family of trigonometric functions based on Sagitta and Cosagitta was used for the description of probabilities of joint detections of entangled particles - Claudius Theorem.

3) Family of trigonometric functions based on Hacoversine was proposed as a new measure for the quantitative determination of gaps between the detections of identical events - David Theorem.

4) David Theorem can be easily tested in Laboratories of Leaders in this field.

\section{Acknowledgments}

This work was supported by the JP\&FŠ Agency (Contract Number 25g/1963), by the VZ\&MŠ Agency (Contract Number 16000/1989) and by the GMS Agency (Contract Number 69110/1992). The valuable inspiration to the Figure 3 was given by CU\&OB from Patria Agency (Contract Number 314/1983). We have found the valuable support on the web site www.wolframalpha.com with the corrections of used formulae.

\section{References}

Aczel, A. D. (2001). Entanglement. The Greatest Mystery of Physics. Four Walls Eight Windows, New York.

Aspect, A. (2015). Viewpoint: Closing the Door on Einstein and Bohr's Quantum Debate. Physics, 8, 123.

Bell, J. S. (1964). On the Einstein-Podolsky-Rosen Paradox. Physics, 1, 195.

Bell, J. S. (2004). Speakable and Unspeakable in Quantum Mechanics. Cambridge University Press, Cambridge.

Bertlmann, R., \& Zeilinger, A. (2017). Quantum [Un] Speakables II. Half a Century of Bell's Theorem. Springer, Swiss.

Bohr, N. (1935). Can Quantum-Mechanical Description of Physical Reality be Considered Complete? Phys. Rev., $48,696$.

Born, M. (1926). Zur Quantemmechanik der Stoßvorgänge (On the Quantum Mechanics of Collisions). In J. A. Wheeler, \& W. H. Zurek (Ed.). Princeton University Press, Princeton. ISBN 0-691-08316-9.

Christensen, B. G. (2015). Exploring Limits of Quantum Nonlocality with Entagled Photons. Phys. Rev., X 5, 041052 . 
Datta, B., \& Singh, A.N. (1983). Hindu Trigonometry. Indian Journal Of History of Science, 18, 39-108. Retrieved from www.insa.nic.in/writereaddata/.../IJHS/Vol18_1_5_BDatta.pdf

Einstein, A., Podolsky, B., \& N. Rosen. (1935). Can Quantum Mechanical Description of Physical Reality Be Considered Complete? Phys. Rev., 48, 696.

Gustina, M. (2015). Significant-Loophole-Freee Test of Bell's Theorem with Entangled Photons. Phys. Rev. Lett., $115,250401$.

Handsteiner, J. (2017). Cosmic Bell Test: Measurement Settings from Milky Way Stars. Phys. Rev. Lett., 118, 060401.

Heisenberg, W. (1989). Encounters with Einstein: And Other Essays on People, Places, and Particles. Princeton University Press, Princeton.

Khrennikov. A. (2016). Quantum and Beyond $(Q B)$. International Conference devoted to quantum theory and experiment. June 13-16, 2016. Retrieved from www.lnu.se/en/staff/andrei.khrennikov video presentations

Kvasz, L. (2008). Patterns of Change. Linguistic Innovations in the Development of Classical Mathematics. Birkhäuser, Basel.

Larsson, J. A. (2014). Loopholes in Bell Inequality Tests of Local Realism. J. Phys. A, 47, 424003. https://doi.org/10.1088/1751-8113/47/42/424003

Maor, E. (1998). Trigonometric Delights. Princeton: Princeton University Press. Retrieved from http://freebooksdl.com/346564-trigonometric-delights-8.html/

Matvievskaya, G. P. (1990). An outline of History of Trigonometry. Tashkent: Fan. Retrieved from $\mathrm{http} / / / \mathrm{www} . y e l l o w p a g e s . u z / c o m p a n y / 11$

Popper, K. (1959). The Logic of Scientific Discovery. New York, NY: Basic Books.

Shalm, L. K. (2015). Strong Loophole-Free Test of Local Realism. Phys. Rev. Lett., 115, 250402.

Smýkalová, R. (2015). Goniometric Fuctions in Elementary Mathematics. Akademické nakladatelství, Brno.

Van Brumelen, G. (2009). The Mathematics of Heavens and Earth (The early history of trigonometry). Princeton: Princeton University Press.

Van Brumelen, G. (2013). Heavenly Mathematics (The forgotten art of spherical trigonometry). Princeton, Princeton University Press.

Van Brummelen, G. (2014). Triangles before Logarithms: Trigonometry in the Lost Century. Retrieved from https://www.youtube.com/watch?v=-OoyZtwhGbY videopresentation.

Von Braumühl, A. (1900). Vorlesungen über Geschichte der Trigonometrie. Leipzig, Teubner. Retrieved from https://archive.org/details/vorlesungenber00brauuoft

\section{Copyrights}

Copyright for this article is retained by the author(s), with first publication rights granted to the journal.

This is an open-access article distributed under the terms and conditions of the Creative Commons Attribution license (http://creativecommons.org/licenses/by/4.0/). 